\title{
El e-portafolio y redes multimedia como herramienta de aprendizaje autónomo. Casos de estudio en Grado y Master.
}

\author{
Modesto Pérez-Sánchez ${ }^{\mathrm{a}}$, Francisco-Javier Sánchez-Romero ${ }^{b}$ P. Amparo López- \\ Jiménez ${ }^{\mathrm{a}}$ \\ ${ }^{a}$ Departamento de Ingeniería Hidráulica y Medio Ambiente. Universitat Politècnica de València. \\ palopez@upv.es; mopesan1@upv.es \\ b Departamento de Ingeniería Rural y Agroalimentaria. Universitat Politècnica de València. \\ fcosanro@agf.upv.es
}

\begin{abstract}
This paper describes the role of the e-portfolio as a tool for a dual purpose: on the one hand, students have to develop problems in an autonomous way, and small case studies based on classroom practices carried out previously. In this sense, the students must apply their problem solving skills. On the other hand, the Poliforma-T platform allows students to register their documents online and to receive feedback for correcting them before the corresponding evaluation. In parallel, the theoretical knowledge is available to the student through learning objects directly related to the content of the problems of study. The methodology has been implemented in two very different subjects: Electrical Engineering Degree, in the topic Fluid Mechanics of the EPSA; and in the topic: Pollutants Dispersion of pollutants in Water and Atmosphere, of Master in Industrial Engineering, with very positive results.
\end{abstract}

Keywords:e-portafolio, Fluid Mechanics, Pollutant Dispersion, autonomous learning, PoliformaT

\section{Resumen}

El presente trabajo describe el papel que juega el e-portafolio como una herramienta que cumple un doble objetivo: de un lado, los alumnos tienen que desarrollar problemas de forma autónoma como pequeños estudios de caso basándose en las prácticas de aula realizadas previamente. En este sentido el alumno debe aplicar sus conocimientos más abstractos a la resolución de problemas. De otro lado, la plataforma Poliforma-T permite al alumno registrar sus documentos en plazos reglamentarios y recibir retroalimentación sobre la corrección de los mismos antes de la evaluación correspondiente. De forma 
paralela, los conocimientos teóricos se encuentran a disposición del alumno a través de objetos de aprendizaje directamente relacionados con el contenido de los problemas de estudio. La metodología se ha implantado en dos asignaturas muy diferentes: Mecánica de fluidos de Grado de Ingeniería Eléctrica de la EPSA; y la asignatura Dispersión de Contaminantes en Agua y en Atmósfera del Máster Universitario en Ingeniería Industrial con unos resultados muy positivos.

Palabras clave: e-portafolio, mecánica de fluidos, dispersión de contaminantes, aprendizaje autónomo, PoliformaT

\section{Introducción}

El desarrollo del personal docente conduce a un proceso en el cual éstos deben revisar, renovar, desarrollar y actualizar las metodologías docentes (Martinez-De la Muela, 2015). El objetivo último ha de ser, facilitar el proceso de aprendizaje del alumno y garantizar el alcance de los resultados de aprendizaje en el marco actual universitario de Espacio Europeo de Educación Superior, permitiendo así alcanzar las necesidades educativas del mismo y conseguir los objetivos propuestos (Barberá, 2005).

En este marco, el portafolio es una herramienta más para conseguir estos resultados de aprendizaje. El concepto del portafolio se refiere al conjunto de evidencias (trabajos, problemas, análisis o actividades similares) recopiladas acerca de una cuestión concreta (Poza-Lujan, 2015). Mediante esta herramienta se puede llevar a cabo un seguimiento del aprendizaje del alumno a lo largo de los contenidos de la asignatura, permitiendo la evaluación de las diferentes unidades didácticas (Barberá, 2008, 2009). Del mismo modo también se puede trabajar y evaluar competencias transversales. Estas competencias son un objetivo estratégico de la Universidad Politécnica de Valencia (Yepes, 2015) que buscan dotar a los titulados de un valor añadido complementario a la formación técnica.

Aunque el portafolio viene utilizándose desde hace décadas (Wiedmer, 1998; Morris, 2000), el desarrollo de las tecnologías puede conducir a mejorar el desarrollo del alineamiento del aprendizaje del alumno (Morris, 2003), máxime si tenemos en cuenta el trabajo no presencial que el nuevo marco actual universitario incorpora a los planes de estudio. Es por ello que en este trabajo se pretende llevar a cabo una evaluación de los resultados obtenidos en dos asignaturas situadas en diferente nivel (primer y segundo ciclo respectivamente) de formación del alumno.

Las asignaturas seleccionadas por parte de los autores han sido, por un lado, "Mecánica de fluidos", impartida en segundo curso del grado de Ingeniería Eléctrica y por otro "Dispersión de contaminantes en agua y atmósfera" impartida en segundo curso de Máster Universitario de Ingeniería Industrial, de primer y segundo ciclo respectivamente. El desarrollo de la experiencia pretende evaluar el éxito de esta técnica en dos asignaturas situadas en dos

(cc) EY-NC-ND 2016, Universitat Politècnica de València 
niveles de formación muy diferentes, en la primera de ellas, al inicio de la formación universitaria y en el segundo caso, finalizando la formación post-grado.

La asignatura de Mecánica de fluidos es una rama dentro de la física de medios continuos que estudia el comportamiento de las sustancias (líquidos y gases) que se deforman siempre y cuando exista una fuerza tangencial provocando un campo de velocidades. Esta asignatura suele encontrarse al inicio de los planes de estudio de ingeniería (segundo o tercer curso) como continuación de los estudios de física y matemáticas sirviendo de base para otras materias curriculares de los estudiantes en cursos superiores. Derivado de su importancia es imprescindible que el alumno alcance los resultados de aprendizaje propuestos en la asignatura para que pueda analizar en profundidad otras asignaturas que en cursos posteriores se sustentarán en los conceptos desarrollados aquí y aplicarlos en el desarrollo de su etapa profesional. La asignatura es obligatoria de 4.5 créditos dentro de la materia Termodinámica y Mecánica de Fluidos. En este curso tiene matriculados 49 alumnos.

De forma completamente opuesta, la asignatura Dispersión de contaminantes en agua y atmósfera es parte de una materia terminal en el Master Universitario de Ingeniería Industrial en la Especialidad en sostenibilidad y medio ambiente industrial como una materia optativa de 4.5 créditos dentro de la Ingeniería de las aguas residuales y de la contaminación atmosférica. Esta asignatura solamente ha tenido seis alumnos en el presente curso y la realización de un eportafolio se propone como una estrategia eficiente en tanto que el aprendizaje es muy directo entre profesor y alumno y los objetivos de la asignatura permiten la implementación de esta estrategia docente.

\section{Objetivos}

El objetivo de la experiencia llevada a cabo es desarrollar, evaluar y mejorar el sistema de evaluación de asignaturas que tradicionalmente han sido teóricas, intentando virar sus sistemas de evaluación a técnicas más dinámicas que ayuden a nuestros alumnos a tener herramientas que favorezcan el aprendizaje profundo en estas materias "huyendo" del aprendizaje superficial que tradicionalmente se ha producido en materias propias de primeros cursos de ingeniería en planes antiguos. El desarrollo de actividades a través del portafolio puede conducir a que el alumno pueda reflexionar sobre los contenidos impartidos, favoreciendo la asimilación y análisis crítico de los mismos. El portafolio permite proporcionar información sobre los resultados tanto al profesor como al estudiante, ya que es una herramienta donde la reflexión del proceso de aprendizaje se une a la exposición de los resultados de la docencia (Olalla, 2015).

En el caso de la asignatura Mecánica de Fluidos, los objetivos planteados ha sido:

- Favorecer la asimilación de los contenidos relativos a la unidad didáctica "Estática de los Fluidos", recogida en el tema 2 de la asignatura, buscando en el alumno, una profundización de los conceptos expuestos en clase.

(c)) EY-NC-ND 2016, Universitat Politècnica de València

Congreso In-Red (2016) 
- Extraer información para poder extrapolar la herramienta del e-portafolio aplicada a esta unidad didáctica, al resto de unidades que forman la asignatura en cursos posteriores. El objetivo es fomentar el trabajo no presencial que tiene establecido la asignatura en el plan de estudios y favorecer el aprendizaje profundo de la asignatura.

- Motivar a los alumnos aplicando los conceptos teóricos de mecánica de fluidos a casos prácticos relacionados con situaciones que pueden darse en el desarrollo de la profesión.

- Establecer mecanismos de comunicación (foro, chat y e-mail) que favorezcan el feedback durante la resolución de las tareas propuestas y posteriormente durante la evaluación.

- Trasladar parte de la evaluación de la asignatura que actualmente se realiza mediante actividades realizadas presencialmente (prácticas de laboratorio y/o informáticas) a actividades no presenciales, fomentando la formación no presencial del alumno.

En el caso de la asignatura de Dispersión de contaminantes en agua y atmósfera los objetivos planteados han sido:

- Favorecer la asimilación de los contenidos relativos a la primera parte de la asignatura en que se ha abordado la dispersión atmosférica de los contaminantes.

- Motivar a los alumnos aplicando los conceptos de asignaturas previas como mecánica de fluidos, tecnologías del medio ambiente y otras en temas terminales como son la determinación de altura de chimenea, la aplicación de modelos gaussianos o el análisis de datos ambientales y climáticos en las diferentes páginas de Internet que se encuentran disponibles gratuitamente.

- Profundizar en aspectos colaterales al desarrollo de la asignatura de forma más aplicada de manera que los alumnos han seleccionado temáticas para presentaciones breves que constituyen el portafolio de los mismos. En cada clase ha habido una o varias presentaciones que motivan a los alumnos más allá de la clase magistral

- Establecer mecanismos de comunicación efectiva, puesto que cada alumno de forma voluntaria ha seleccionado temas concretos profundizando en el temario, y ha hecho una exposición pública de los mismos con una duración de menos de diez minutos en castellano o en inglés, recibiendo en el mismo idioma preguntas de sus compañeros.

- Conocer en profundidad temas de gran aplicación y actualidad derivados de los conceptos presentados en la asignatura que han dirigido a algunos de los alumnos a seleccionar sus Trabajos final de máster en la temática de la asignatura.

(cc) EY-NC-ND 2016, Universitat Politècnica de València 


\section{Desarrollo de la innovación}

La innovación en este campo va a ser la adaptación de la herramienta del e-portafolio a ambas asignaturas en los conceptos descritos anteriormente, con el objetivo de profundizar en los conceptos explicados en la clase de teoría de aula, con ayuda de objetos de aprendizaje y material complementario dejado en la plataforma de Poliformat. Esta es una herramienta de "campus virtual" basada en el proyecto "Sakai".

El objetivo no es desarrollar una actividad que el alumno la vea como un trabajo "extra" sino se pretende que, con ayuda de las herramientas informáticas, fomentar el trabajo continuo del alumno (no presencial) a través de tareas propuestas en el Poliformat. Estas tareas activadas a lo largo de la unidad buscan constituir un compendio de estrategias que permitan la reflexión del alumno de los contenidos teóricos desarrollados en la unidad; autoevaluarse su propio proceso de aprendizaje y encontrar un medio que motive a un conocimiento más profundo del alumno por los temas tratados.

En los casos presentados en este artículo esta reflexión será de forma diferente. En el caso de Mecánica de Fluidos la reflexión viene apoyada en la resolución de ejercicios propuestos aplicados a situaciones cotidianas, en las que el alumno busca poner la evidencia del fenómeno de las presiones hidrostáticas aplicados sobre superficies. En el segundo caso se buscan evidencias seleccionas por los propios alumnos como resultado de la reflexión sobre su propio proceso de aprendizaje de forma que con la presentación de breves conceptos se profundice en temas que son adyacentes a la asignatura, pero de gran aplicabilidad.

En el caso de la asignatura de Mecánica de Fluidos, tal y como se ha citado anteriormente, únicamente ha sido desarrollada en una (Estática de los fluidos) de las siete unidades didácticas que constituyen la asignatura. La metodología llevada a cabo ha sido:

1) Desarrollo teórico en aula de los conceptos teóricos pertenecientes a la estática de los fluidos y aplicación práctica, que de acuerdo a lo recogido en la guía docente tiene una dedicación presencial de 5 horas (metodología tradicional).

2) Realización de prácticas de aula donde el alumno realiza casos prácticos sencillos de aplicación de las ecuaciones generales de la hidroestática (metodología tradicional), con una dedicación presencial de $2 \mathrm{~h}$.

3) Al mismo tiempo que se desarrollan los puntos 1) y 2) anteriormente citados, a través del Poliformat se activan tareas con el objetivo de completar el e-portafolio de forma continúa fomentando el proceso de aprendizaje profundo. Los diferentes apartados del e-portafolio (cumplimentado en las tareas propuestas) tienen un tiempo preestablecido de realización, con la finalidad de que el alumno no desatienda el trabajo no presencial de la asignatura en esta unidad, que en este caso está valorado en 14 horas en la guía docente.

4) Las tareas a completar que forman el e-portafolio se abren en dos ventanas temporales tras el desarrollo teórico presencial. Una primera parte, correspondiente a la ecuación general de la hidroestática, en la que el alumno desarrolla y profundiza sobre las presiones ejercidas por un fluido o varios en el caso de estar estratificado

(c)) EY-NC-ND 2016, Universitat Politècnica de València

Congreso In-Red (2016) 
y su utilización en el campo de la mecánica de fluidos para la realización de medición de presiones manométricas mediante piezómetros y manómetros. La segunda parte del e-portafolio, se abre en Poliformat una vez desarrollada la teoría de fuerzas hidroestáticas sobre superficies planas y curvas. Esta segunda fase permite al alumno reflexionar sobre los conceptos y conocimientos ya aplicados en la primera parte del portafolio, actuando por un lado de repaso de los contenidos ya desarrollados $\mathrm{y}$, por otro lado, aplicar estos conceptos a ejemplos prácticos relacionados con su futuro profesional. Este hecho les conduce a aumentar su motivación al ver un sentido práctico a los contenidos teóricos desarrollados.

5) De forma paralela al desarrollo de las actividades abiertas en el apartado de Tareas del Poliformat que constituyen el e-portafolio se establece un canal "online" de resolución de dudas lo que facilita al alumno la resolución de dudas e inquietudes sobre los conceptos con el profesor. Del mismo modo se pone a disposición del alumno, objetos de aprendizaje relacionados con la unidad didáctica que fragmentan los contenidos de la unidad y les ayudan a adquirir los conceptos expuestos en clase al ritmo de cada uno.

6) Al entregar la primera parte de la actividad propuesta en la unidad se procede a la corrección de la misma, devolviendo los resultados y correcciones antes de abrir la segunda parte de la actividad. Esto facilita al alumno un feedback directo de los resultados de aprendizaje alcanzados en la primera parte, pudiendo corregir y profundizar en caso de que le sea necesario antes de comenzar la siguiente actividad.

7) Los resultados de la segunda parte son devueltos en la semana siguiente, con las correcciones y comentarios sirviendole de retroalimentación y aplicación en casos prácticos de las siguientes unidades didácticas.

8) Para motivar la realización del e-portafolio, aunque son trabajos no presenciales, se ha optado por dar un peso del $10 \%$ sobre la nota final (5\% cada parte propuesta), con el objeto de que el alumno vea recompensado su esfuerzo de realizar el seguimiento continuo de la unidad de acuerdo a lo establecido al nuevo marco de estudios.

La Metodología Docente propuesta en la asignatura Dispersión de contaminantes en agua y atmósfera se propone para alumnos mucho más maduros, que se encuentran en una fase muy terminal de su formación y que deben presentarse en un mercado laboral altamente especializado en muy poco tiempo, por lo que la propuesta sigue la siguiente metodología:

1) Desarrollo en el aula de los contenidos de la asignatura.

2) Cada tema implica una lluvia de ideas sobre conceptos relacionados que se propone realizar terminada cada clase teórica

3) De las ideas relacionadas, los alumnos escogen algunas de ellas para presentarlas en las clases de la semana siguiente, de forma voluntaria.

4) Las presentaciones se realizan al principio de las siguientes clases, en castellano o inglés y se realiza una tanda de preguntas por parte de todos los compañeros. Todos los alumnos deben realizar preguntas en cada tema igualmente en castellano o inglés

(cc) EY-NC-ND 2016, Universitat Politècnica de València

Congreso IN-RED (2016) 
5) Terminada la presentación se realiza una crítica sobre la exposición y cómo el alumno la ha expuesto, haciendo hincapié en los puntos fuertes y débiles de cada uno

6) Las presentaciones se almacenan en un lugar común para los alumnos en la herramienta de PoliformaT, de manera que pueden consultarse y comentarse para aquellos alumnos que no han podido asistir al debate

7) Cada presentación voluntaria se evalúa con 0,25 puntos sobre la nota final. Cada alumno puede hacer tantas como desee. Todos los alumnos deben hacer al menos una de estas disquisiciones orales voluntarias.

El acceso de los alumnos a sus presentaciones y los comentarios críticos sobre las mismas, volcados en el e-portafolio, favorece la reflexión sobre su propia capacidad de comunicación, convirtiéndose en una evidencia, a su vez, de la adquisición de la competencia transversal "Comunicación Efectiva", de la que se es punto de control.

\section{Resultados}

Los resultados obtenidos en la asignatura de mecánica de fluidos han sido muy satisfactorios. En primer lugar, ha habido una participación del $95.6 \%$ en la primera actividad y un $91.8 \%$ en la segunda actividad en las actividades lo que pone de manifiesto la buena acogida por parte de los alumnos. En cuanto a los resultados obtenidos en la corrección de los ejercicios, se adjunta en la Tabla 1, donde un índice de aprobados del $85.1 \%$ y $82.2 \%$. Si comparamos estos resultados, con los obtenidos de los mismos ejercicios propuestos en exámenes de la misma asignatura en otros grados, con el mismo profesorado, presentan tasas de éxito del orden un $30 \%$ menor, próximo al 50\%. Se tiene que tener en cuenta, que las actividades están individualizadas para cada uno de los alumnos, variando los datos de los problemas en función del número de su Documento Nacional de Identidad.

Tabla 1. Resultados obtenidos en la evaluación de las actividades propuestas en mecánica de fluidos

\begin{tabular}{ccc}
\hline Nota Obtenida & Primera Actividad & Segunda Actividad \\
\hline $\mathbf{9 - 1 0}$ & 5 & 3 \\
$\mathbf{7 - 8 . 9 9}$ & 15 & 19 \\
$\mathbf{5 - 6 . 9 9}$ & 20 & 15 \\
$\mathbf{3 - 4 . 9 9}$ & 7 & 7 \\
$\mathbf{0 - 2 . 9 9}$ & 0 & 1 \\
No realizada & 2 & 4 \\
\hline
\end{tabular}

Además de los buenos resultados obtenidos, en cuanto a participación y evaluación de la actividad, durante las tres semanas que se impartió esta unidad didáctica los alumnos realizaron un total de 19 y 16 consultas via email (una media de 12 consultas por semana). Este hecho muestra el trabajo no presencial de los alumnos, comparado con la media en años anteriores en la misma asignatura (incluso en otros grupos) en que no suelen existir más de 1 o 2 solicitudes de tutorías o en el mismo curso en las unidades que no se ha planteado esta metodología. Este hecho pone de manifiesto que el desarrollo de la actividad ha propiciado el efecto que se buscaba: fomentar el trabajo no presencial en el aula y favorecer la

(c)) EY-NC-ND 2016, Universitat Politècnica de València

Congreso In-Red (2016) 
asimilación de los contenidos desarrollados, así como motivar a los alumnos a profundizar en los conceptos abordados en las sesiones de aula

En cuanto a la asignatura de Dispersión de contaminantes en agua y atmósfera, los resultados son diferentes, ya que los alumnos son muchos menos y la distinta naturaleza de los objetivos. En este caso han participado la totalidad de los alumnos y se han abordado las siguientes temáticas en las diez últimas de las doce sesiones en que se ha impartido la clase:

- La contaminación atmosférica y la capa de ozono

- La lluvia ácida

- El efecto invernadero y el protocolo de Kioto

- Internet sites for collecting metheorological and air quality data

- Industrial chimneys: matherials, tipologies, examples

- Parámetros de control de la contaminación atmosférica

- Instrumentos de medición de parámetros meteorológicos

- Eventos graves de contaminación atmosférica a lo largo del tiempo

- Air quality models available in Internet: An overview

- Fenómenos meteorológicos a gran escala: El Niño y La Niña

\section{Conclusiones}

En las dos experiencias llevadas a cabo de la utilización del e-portafolio se ha puesto de manifiesto el éxito en cada una de ellas, favoreciendo el aprendizaje profundo de los alumnos participantes a través de la reflexión y análisis crítico de actividades propuestas. En ambos casos se ha registrado una alta participación de los alumnos, lo que refleja la buena acogida por parte de los estudiantes a la realización de las tareas del portafolio, poniendo en valor los aspectos en que coinciden y aquellos en los que se diferencian.

En el caso de Mecánica de fluidos, en cursos siguientes se va a introducir este sistema de trabajo en otras unidades didácticas de la asignatura, con el fin de fomentar el trabajo no presencial y motivar al alumno en este tipo de asignaturas con un carácter tan teórico, pero de gran importancia en futuras asignaturas de cursos posteriores.

La utilización del e-portafolio junto con objetos de aprendizaje pueden resultar básicos para guiar al alumno en el proceso de aprendizaje, pudiendo éste establecerse su propio ritmo de aprendizaje. La utilización de las herramientas informáticas y sobretodo el Poliformat, resultan fundamentales para: ayudar al alumno con actividades propuestas por parte del profesor, ayudar al profesor a recoger evidencias que permitan la evaluación de contenidos impartidos y establecer una retroalimentación alumno-profesor-alumno que permita alcanzar con mayor éxito los resultados de aprendizaje.

La diferencia con la motivación, la madurez personal y las competencias adquiridas en cuanto a habilidades para la comunicación y el aprendizaje autónomo de los alumnos a través de la preparación de esas pequeñas presentaciones, hacen que la asignatura Dispersión de

(cc) EY-NC-ND 2016, Universitat Politècnica de València

Congreso IN-RED (2016) 
Contaminantes en Agua y Atmósfera haya tenido resultados diferentes pero no menos satisfactorios: la totalidad de los alumnos han realizado al menos una presentación, sino dos, en relación con una temática que ha despertado su interés, se ha presentado en público, criticado y participado activamente en las temáticas de los demás. Ello ha infundido en los alumnos gran interés y afán por realizar cada vez mejores presentaciones orales, mejorando así su capacidad de comunicación oral efectiva, favoreciendo las consultas de tutorías al profesor y permitiendo que el e-portafolio se convierta en un espacio que aúna todas las evidencias combinando el trabajo presencial y no presencial de un aprendizaje más profundo por parte de alumnos de primeros y últimos cursos en materias curriculares bien diversas como son las aquí presentadas.

El diseño de nuevas actividades, apoyadas en los recursos que nos presentan las TIC's actuales, pueden ser herramientas que permitan alcanzar los resultados de aprendizaje en materias históricamente consideradas muy teóricas, introduciendo al alumno en entornos de trabajo que le motiven y le hagan mantener una actitud activa en el seguimiento de la misma. El aprovechamiento de estas herramientas permitirá aumentar el número de actividades productivas frente a las actividades asimilativas que predominan en las carreras técnicas.

\section{Referencias}

BARBERÁ, E. (2005). La evaluación de competencias complejas. La práctica del portafolio. EDUCERE. Revista Venezolana de Educación,.(31). pp.497-503.

< http://www.saber.ula.ve/browse-title> /> [Consulta:19 de Mayo de 2016]

BARBERÁ, E. (2008). El estilo e-portafolio. Barcelona: UOC.

BARBERÁ, E., GEWERC BARUJEL, A. y RODRÍGUEZ ILLERA, J.L. (2009). "Portafolios electrónicos y educación superior en España: Situación y tendencias". RED, Revista de Educación a Distancia. Número monográfico VIII, pp.1-13. 〈http://www.um.es/ead/red/M8/> [Consulta:13 de Marzo de 2016]

BARRAGÁN, R. (2005). El portafolio metodología de evaluación y aprendizaje de cara al nuevo espacio de educación superior. Una experiencia práctica en la universidad de Sevilla. Revista Latinoamericana de Tecnología educativa, 4(1), pp. 121-139.

Martínez-De la Muela, A.; García-García, M. y Arteaga-MartíneZ, B. "El eportfolio como herramienta para el desarrollo profesional docente". I Congreso Nacional de Innovación Educativa y de Docencia en Red (Valencia, 30 de junio y 1 de julio, 2015). Editorial Universitat Politècnica de València, 2015 Ref.: 6257_01_01_01. DOI: http://dx.doi.org/10.4995/INRED2015.2015

Morris, J.; BuCKLAND, H. (2000). Electronic Portfolios for Learning and Assessment. Proceedings: Society for Information Technology and Teacher Education (SITE) San Diego CA, pp. 1085-1090.

Morris, J. (2003). Digital Portfolios Reflect How We Learn. In C. Crawford, N. Davis, J. Price, R. Weber \& D. Willis (Eds.), Proceedings of Society for Information Technology \& Teacher Education International Conference 2003 (pp. 122-127)

(c)) EY-NC-ND 2016, Universitat Politècnica de València

Congreso In-Red (2016) 
Olalla, A. G. (2015). El portafolio docente. Un instrumento para evaluación y mejora de la práctica docente. Revista del Congrés Internacional de Docència Universitària i

Innovació

(CIDUI), (2).

Poza-Lujána J.L.; Cabrera Mendez; M.; Rebollo, M.; Calduch, A.; DiezSomavilla, R.; Lloret Romero, N.; Despujol Zabala, I.; Albors, A.; Teruel, L. "Experiencia en el uso del portafolio por medio de las redes sociales". I Congreso Nacional de Innovación Educativa y de Docencia en Red (Valencia, 30 de junio y 1 de julio, 2015). Editorial Universitat Politècnica de València, 2015 Ref.: 6257_01_01_01. DOI: http://dx.doi.org/10.4995/INRED2015.2015

WIEDMER, T. L. (1998). Digital portfolios. Phi Delta Kappan, 70 (8), 586-590.

YEPES, V.; MARTÍ, J.V. "La competencia transversal de comunicación efectiva en estudios de máster en el ámbito de la ingeniería civil y la construcción”. I Congreso Nacional de Innovación Educativa y de Docencia en Red (Valencia, 30 de junio y 1 de julio, 2015). Editorial Universitat Politècnica de València, 2015 Ref.: 6257_01_01_01. DOI: http://dx.doi.org/10.4995/INRED2015.2015 